\title{
Worldwide collaboration for orphan drug designation
}

\author{
Segundo Mariz, James H. Reese, Kerstin Westermark, Lesley Greene, \\ Takahiro Goto, Tatsuro Hoshino, Jordi Llinares-Garcia and Bruno Sepodes
}

The growing awareness of the need for pharmaceutical products to treat rare diseases led to the enactment of rare diseases legislation in the United States (Emergence of orphan drugs in the United States: a quantitative assessment of the first 25 years. Nat. Rev. Drug Discov. 9, 510-522 (2010) $)^{1}$, the European Union ${ }^{2}$ and $\operatorname{Japan}^{3}$ (see also Further information). The European Medicines Agency (EMA) has been engaged in collaborations with the US Food and Drug Administration (FDA)'s Office of Orphan Products Development (OOPD) since 2000 and with the Japanese Ministry of Health, Labour and Welfare (MHLW) and Pharmaceuticals and Medical Devices Agency (PMDA) Orphan Drug Designation Service since 2010. Identifying areas of similarity among these programmes has led to activities aimed at reducing redundancies and the administrative load for sponsors interested in submitting applications for orphan drug designation in each region, allowing for transparency and an increased sponsor understanding of each agency's processes and incentives (TABLE 1). It is hoped that there will be an increase in awareness of the benefits of parallel submission both at the orphan designation stage and post-designation, where input through protocol assistance (scientific advice) and paediatric input are subject to incentives. The reduction in regulatory consultation times and improved efficiencies in drug development due to these collaborative efforts should translate into accelerated timelines for the approval of successful orphan drugs.

\section{EMA-FDA collaboration}

In 2007, a joint application form for orphan drug submissions in the United States and the European Union was introduced, reducing the sponsor's administrative burden and encouraging parallel submissions to both agencies. The agencies have worked together to understand areas of similarity at the time of submission for an orphan drug designation, enhancing the understanding of key assessment criteria, which are similar in both systems. The OOPD Drug Designation Program carries out assessments for the FDA, whereas in Europe this is done by the Committee for Orphan Medicinal Products (COMP).
Definition of an orphan condition. The sponsor often plans for development of a drug for a specific therapeutic indication that falls within an orphan disease or condition. Therefore, often the overall orphan disease or condition may be broader than the therapeutic indication that the sponsor plans to study for marketing approval. Although there is some variance between the interpretations by both agencies $^{4,5}$, there has been an effort by both parties to understand the other's approach to setting the boundaries of a rare disease or condition at the time of designation. They also aimed to produce less variance in the review approach for the same product when a sponsor seeks a designation at the EMA and FDA. Through this type of collaboration, the post-designation clinical development may be more focused, thereby increasing efficiencies in development and the probability of success. The agencies also consult each other regarding changes in classifications or definitions of orphan conditions that have occurred as a consequence of advances in science.

Medical plausibility and scientific rationale. Both agencies require sponsors to submit data to support the hypothesis that the product has a mode of action that has sound pharmacological principles regarding the product's potential use in the condition ${ }^{6}$. Preliminary surveys conducted independently by both agencies show that $\sim 30 \%$ of submissions that have demonstrated acceptable medical plausibility use preclinical in vivo data, and the remainder generally have promising preliminary clinical data with the product in patients with the condition (FIG. 1a). Only a very small percentage of acceptable medical plausibility sections have preclinical data limited to in vitro data ( $2 \%$ for both the FDA and EMA) (FIG. 1b). This scientific rationale prerequisite has been identified as necessary in establishing an orphan designation in both systems, and similar datasets can be used in a sponsor's submission.

Prevalence. The US legislation defines a 'rare disease or condition' as a disease or a condition that affects fewer than 200,000 people in the United States. In the European Union, the orphan regulation establishes that the prevalence has to be based on epidemiological data from the European Economic Area (EEA; consisting of the 28 EU Member States in addition to Norway, Liechtenstein and Iceland) and must not be more than 5 in 10,000 people. In the United States, the number corresponds to a higher prevalence rate (around 7 in 10,000) than in Europe, which means that certain conditions may obtain an orphan designation in the United States but do not qualify in Europe.

Procedural aspects. Text used to describe the rare disease or condition and the medical plausibility and scientific rationale will not vary much in view of the similarity of the requirements, making the only different task the prevalence calculation. In the European Union, once a submission is validated, a start date is given and the procedure lasts a maximum of 90 days with no clock-stop period permitted. There is no time limit specified in the law or in FDA regulation within which the FDA must respond to a submission request, although the FDA OOPD works to provide sponsors with a timely response.

In the European Union, there is the additional requirement of conducting a search for other pharmaceutical medicinal products authorized for use in the orphan condition and the need to establish, where necessary, the basis for the assumption of significant benefit. Significant benefit is defined either as a clinically relevant advantage or a major contribution to patient care that the product may give. Additionally, the EMA requests that sponsors explain the current status of the product development programme as well as regulatory considerations associated with orphan drug designation.

Both agencies have worked to enhance the transparency of the administrative requirements on their websites. In 2011, both agencies agreed that it was acceptable to receive annual reports using the template provided through the EMA and available on the EMA orphan web section, reducing the need for duplication and instead focusing on the information, which was relevant to both agencies.

Topics also under consideration for further consultation are the data requirements for the principles of clinical superiority and major contribution to patient care. The FDA and EMA have established a parallel scientific advice procedure, which includes products that have obtained an orphan designation. Parallel scientific advice offers the possibility of resolving questions about clinical data needs, thereby offering the potential of making clinical development programmes more efficient and reducing times from submission to approval. 
Table 1 | Comparison of incentives for products that are granted orphan drug designation

\begin{tabular}{|c|c|c|c|}
\hline & United States & European Union & Japan \\
\hline $\begin{array}{l}\text { Financial } \\
\text { incentives }\end{array}$ & $\begin{array}{l}\text { - Tax credits can apply to as much } \\
\text { as } 50 \% \text { of qualified clinical } \\
\text { development costs (US studies) } \\
\text { - User fees paid to the FDA } \\
\text { for review of the sponsors' } \\
\text { application for marketing } \\
\text { authorisation are waived }\end{array}$ & $\begin{array}{l}\text { - No general tax credit on clinical trials and no } \\
\text { specific subsidies for clinical trials } \\
\text { - Regulatory fee reductions generally favour } \\
\text { small and medium-sized enterprises, but are } \\
\text { revised from time to time } \\
\text { - Member states might offer a variety of price } \\
\text { and reimbursement incentives as well as tax } \\
\text { credits (see REF. 7) }\end{array}$ & $\begin{array}{l}\text { - Financial subsidies for up to } 50 \% \text { of } \\
\text { expenses for clinical and non-clinical } \\
\text { research } \\
\text { - Subsidies through the NIBIO to reduce the } \\
\text { financial burden of product development } \\
\text { - User fee waivers, } 15 \% \text { tax credits, up to } \\
20 \% \text { corporate tax reduction and a 30\% } \\
\text { reduction in marketing application fees }\end{array}$ \\
\hline $\begin{array}{l}\text { Grants for } \\
\text { research } \\
\text { programmes }\end{array}$ & $\begin{array}{l}\text {-The FDA Orphan Products } \\
\text { Grant Program offers funding } \\
\text { for clinical studies (investigating } \\
\text { safety and/or effectiveness) that } \\
\text { will result in or substantially } \\
\text { contribute to market approval } \\
\text { - The National Institutes of } \\
\text { Health (NIH) also has a grants } \\
\text { mechanism for rare diseases }\end{array}$ & $\begin{array}{l}\text { - The European Commission supports rare } \\
\text { disease research through its framework } \\
\text { programmes and the call for proposals } \\
\text { in the rare disease area usually includes } \\
\text { Europe-wide studies of the natural history } \\
\text { of rare disease, pathophysiology and the } \\
\text { development of preventive, diagnostic and } \\
\text { therapeutic interventions } \\
\text { - Member states offer a variety of grants } \\
\text { (see REF. 7) }\end{array}$ & $\begin{array}{l}\text { - Support measures include grants in aid for } \\
\text { clinical and non-clinical research programs, } \\
\text { price-control policies negotiated by } \\
\text { Japanese National Health Insurance and } \\
\text { pharmaceutical companies, and medical } \\
\text { expense reimbursement for } 56 \text { diseases } \\
\text { - NIBIO and AMED offer grant programmes } \\
\text { to small and medium-sized enterprises and } \\
\text { researchers who are developing products } \\
\text { for rare diseases }\end{array}$ \\
\hline
\end{tabular}

AMED, Agency for Medical Research and Development; FDA, US Food and Drug Administration; NIBIO, National Institute of Biomedical Innovation.

Joint annual workshops were established in 2011 at which both agencies present the basis of their orphan designation systems and considerations for post-designation processes.

It has been noted that currently $~ 50 \%$ of submissions to the EMA are done in parallel with the FDA, with $\sim 30-40 \%$ of applicants using the joint FDA-EMA application form. Although the review processes are done independently, most sponsors obtain their respective orphan designation in both regions within a maximum of 6 months of each other.

EMA collaboration with the MHLW/PMDA The EMA's collaboration with the MHLW/ PMDA began in late 2010. Two of the aims of this collaboration are the creation of greater mutual awareness of the submission processes for orphan medicine designations and the development of a system of exchange regarding the outcomes of orphan designations.

Due to the administrative differences between the EMA and the MHLW/PMDA, a common designation application form was not considered feasible. All submissions to Japanese authorities have to be submitted in Japanese. The EMA and the MHLW/PMDA have worked together to establish greater clarity regarding each other's processes in the hope of encouraging parallel submissions and facilitating access to mutual incentive programs for orphandesignated products. An English website for the MHLW was developed, and the EMA Orphan Designation website has some links to the MHLW's website. The MHLW also provides consultations that help sponsors to understand the regulatory process and administrative needs regarding submission requirements.
The MHLW is primarily involved in administrative steps regarding orphan designation, and assessment work is performed primarily by the PMDA. The Pharmaceutical Affairs and Food Sanitation Council (PAFSC) discuss the assessment provided by the PMDA. The PAFSC makes the recommendation to the MHLW on whether to grant a designation, and the MHLW officially grants it. There is no time limit for the assessment process and it is similar to the FDA assessment process. One similarity between the Japanese and the European systems is the use of a committee of independent experts to make a recommendation for granting a designation.

Definition of an orphan condition. The MHLW/PMDA require similar considerations to those requested by the EMA when describing the orphan condition in an application ${ }^{3}$. 

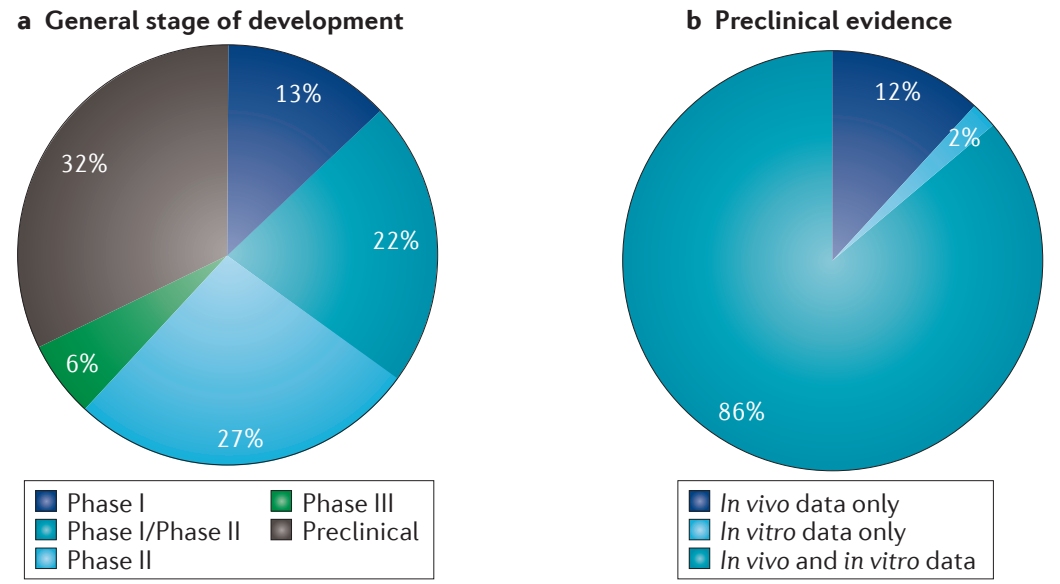

Figure 1 | Information in applications for orphan drug designation in the European Union from 2000 to 2014. These charts are based on internal European Medicines Agency (EMA) data derived from the 1,406 applications for orphan designation that were granted a positive opinion by the Committee for Orphan Medicinal Products (COMP) and orphan designation by the European Commission between 2000-2014. a | The general stage of development for the products for which applications have been submitted for orphan designation. The analysis of the level of development of an orphan drug at the time of designation by the COMP suggests that the majority of designations are based on clinical data. Nevertheless, a substantial percentage of applications comprise preclinical data only. $\mathbf{b} \mid$ The level of preclinical evidence in the applications that were submitted.

Applicants may consider submitting similar information used in a European submission when submitting in Japan. This will benefit post-designation considerations regarding product development and scientific advice, which is conducted by the PMDA, by providing a better understanding of how an orphan condition is defined in both Europe and Japan.

Medical plausibility. Some of the evidence of medical plausibility used in a European submission may also be used in a Japanese submission. There is a difference between the two systems concerning the level of evidence required to support the medical plausibility. In Europe, both preclinical data in a valid in vivo model and/or preliminary clinical data are accepted as the basis for supporting the hypothesis that the product holds promise for the condition. In Japan, the MHLW/PMDA require a higher level of evidence for the purpose of assessment of medical plausibility, as a sponsor should present data from clinical studies to support medical plausibility.

Prevalence. The prevalence threshold in Japan is different to the one required in Europe, and it is similar to the FDA's in that it is based on a number of patients rather than a percentage. The number of patients who may require an orphan drug should be less than 50,000 in Japan, and this calculation must be based on epidemiological data from Japan. Additionally, the sources that are acceptable to the MHLW/ PMDA should be discussed with them at the consultation meeting.
The MHLW/PMDA request applicants to demonstrate that there are high medical needs satisfying one of the following criteria: there is no appropriate alternative drug or treatment, and high efficacy or safety is expected compared with existing products. So, the scientific submission section in Japan has similarities with a European submission. Such similarities in format and criteria could help to reduce the administrative burden for sponsors considering submission to the two systems.

Japan participated in the latest Joint Orphan Workshop with the FDA and the EMA, which was held in London, UK, in March 2014. These meetings allowed sponsors to discuss regulatory considerations in Japan and Europe as well as incentive programmes, with the hope that this will help to create greater efficiencies in post-designation product development and marketing authorisation submissions. The EMA has had discussions with the PMDA regarding similarities and differences in the scientific advice process regarding products that have obtained an orphan designation.

\section{Conclusion}

Many countries have introduced a combination of regulations and policies for orphan drugs in the last two decades. Through parallel submissions and greater transparency of incentive programmes, having early and frequent interactions with regulators, it is hoped that a more global approach to the development of orphan medicines can be fostered, ultimately leading to greater benefits for patients with rare diseases.
Segundo Mariz and Jordi Llinares-Garcia are at the European Medicines Agency, 30 Churchill Place, Canary Wharf, E14 5EU London, UK.

James H. Reese is at the Office of Orphan Products Development, US Food and Drug Administration, Silver Spring, Maryland 20993, USA.

Kerstin Westermark is at the Medical Products Agency, Box 26, SE-75103 Uppsala, Sweden.

Lesley Greene is at the European Medicines Agency, 30 Churchill Place, Canary Wharf, E14 5EU London,

UK; and European Organisation for Rare Diseases (Eurordis), 96 rue Didot, 75014 Paris, France.

Takahiro Goto and Tatsuro Hoshinoare at the Evaluation and Licensing Division, Pharmaceutical and Food Safety Bureau (PFSB), Ministry of Health, Labour and Welfare (MHLW), 1-2-2 Kasumigaseki Chiyoda-ku, Tokyo, 100-8916 Japan.

Bruno Sepodes is at the European Medicines Agency, 30 Churchill Place, Canary Wharf, E14 5EU London, UK; and Universidade de Lisboa, Faculdade de Farmácia, 1649-003 Lisboa, Portugal. Correspondence to B.S. bsepodes@ff.ulisboa.pt doi: 10.1038/nrd.2016.80 Published online 1 Jun 2016; corrected online 15 Jul 2016

Braun, M. M. et al. Emergence of orphan drugs in the United States: a quantitative assessment of the first 25 years. Nat. Rev. Drug Discov. 9, 519-522 (2010). Westermark, K. et al. European regulation on orphan medicinal products: 10 years of experience and future perspectives. Nat. Rev. Drug Discov. 10, 341-347 (2011). Song, P., Gao, J., Inagaki, Y., Kokudo, N. \& Tang, W. Rare diseases, orphan drugs, and their regulation in Asia: current status and future perspectives. Intractable Rare Dis. Res. 1, 3-9 (2012).

4. [No authors listed]. Orphan Drug Act. H. R. 5238 , Public Law No. 97-414, 97th Congress. National Institutes of Health https://history.nih.gov/research/ downloads/PL97-414.pdf (1983).

5. European Commission. Guideline on the format and content of applications for designation as orphan medicinal products and on the transfer of designations from one sponsor to another. European Commission: Health and Consumers Directorate-General http://ec. europa.eu/health/files/orphanmp/2014-03_guideline_ rev4_final.pdf (2014).

6. Tsigkos, S. et al. Establishing medical plausibility in the context of orphan medicines designation in the European Union. Orphanet J. Rare Dis. 9, 175-178 (2014).

7. European Commission. Inventory of Union and

Member State incentives to support research into, and the development and availability of, orphan medicinal products. European Commission: Health and Food Safety http://ec.europa.eu/health/files/orphanmp/doc/ orphan_inv_report_20160126.pdf (2015).

Competing interests statement

The authors declare no competing interests.

Disclaimer

The views expressed in this article are the personal views of the authors and may not be understood or quoted as being made on behalf of, or reflecting the position of, the European Medicines Agency (or one of its committees or working parties), the US Food and Drug Administration or the Japanese Ministry of Health Labour and Welfare and the Pharmaceuticals and Medical Devices Agency of Japan.

\section{FURTHER INFORMATION}

European Medicines Agency: http://www.ema.europa.eu/ ema/index.jsp?curl=pages/regulation/general/general content 000029.jsp\&mid=WCOb01ac05800240ce US Food and Drug Administration: http://www.fda.gov/ Forlndustry/DevelopingProductsforRareDiseasesConditions/ ucm2005525.htm

Japanese Ministry of Health, Labour and Welfare and Pharmaceuticals and Medical Devices Agency: http://www. mhlw.go.jp/english/policy/health-medical/pharmaceuticals/ orphan drug.html

ALL LINKS ARE ACTIVE IN THE ONLINE PDF 